\title{
Fluorescence analysis of urine and its potential for ovarian cancer screening
}

\author{
D. MARTINICKY ${ }^{1, *}$, M. ZVARIK ${ }^{2}$, L. SIKUROVA ${ }^{2}$, I. LAJDOVA ${ }^{3}$, L. HUNAKOVA ${ }^{4}$
}

${ }^{1}$ Department of Gynecological Oncology, National Cancer Institute, Bratislava, Slovakia; ${ }^{2}$ Department of Nuclear Physics and Biophysics, Faculty of Mathematics, Physics and Computer Science, Comenius University, Bratislava, Slovakia; ${ }^{3}$ Department of clinical and experimental pharmacotherapy, Slovak medical university, Bratislava, Slovakia; ${ }^{4}$ Cancer Research Institute, Slovak Academy of Sciences, Bratislava, Slovakia

*Correspondence: david.martinicky@yahoo.com

Received November 10, 2014 / Accepted December 17, 2014

\begin{abstract}
Early diagnosis of ovarian cancer could lead to decreased mortality. We assessed the possible use of urine autofluorescence analysis in its diagnostics and screening.

We analysed urine from 42 healthy volunteers, 35 patients with benign, and 36 patients with malignant ovarian tumors. Synchronous fluorescence spectra with a $70 \mathrm{~nm}$ wavelength difference were recorded for $(1: 1-1: 1024)$ urine dilutions. Concentration matrices of synchronous spectra (CMSS) were used to classify samples into tested groups.

CMSS analysis allowed us to distinguish patients with malignant tumors from healthy ones with a high sensitivity (91.67\%) and specificity (100\%), a positive predictive value (PPV) $100 \%$ and a negative predictive value (NPV) $93.33 \%$. However, discrimination between benign and malignant ovarian tumors was weaker, with sensitivity $86.11 \%$, specificity $77.14 \%$, PPV $79.49 \%$ and NPV $84.38 \%$. Fluorescence intensity and the position of peaks at 330 and $360 \mathrm{~nm}$ were found to be associated with the grade and stage, suggesting that different fluorescent metabolites may prevail at different stages of the disease.

CMSS analysis of urine provides an alternative for ovarian cancer screening method development and could be used as a diagnostic test to detect the recurrence of the disease after therapy.
\end{abstract}

Key words: adnexal tumor, metabolite, native fluorophore, ovarian cancer, synchronous fluorescence spectrum, urine

In 2012, the estimated incidence, mortality and 5-year prevalence of ovarian cancer in women in the world was 238719,151905 and 586624 respectively [1]. Approximately three quarters of cases present in the advanced stages (stages III and IV) where the 5-year overall survival (OS) is $18-34 \%$ in contrast to $66-89 \%$ in early stages (stage I and II) [2]. Diagnosis in the early stages could lead to decreased mortality through early curative therapy [3]. At present there are no screening strategies with proven efficacy for the early detection of ovarian cancer, although several screening studies have been completed or are in progress $[4,5,6,7]$.

Urine is an aqueous solution of a variety of organic and anorganic compounds, including a number of natural fluorophores, most of which are metabolites of tryptophan $[8,9]$. These substances cause a strong blue-green fluorescence of urine. The concentration of urine fluorophores can vary depending on dietary intake, body metabolism, sex and age [10]. Some diseases which are accompanied by metabolic disorders may be associated with the appearance of additional organic compounds in the urine. These are often used as additional indicators in the diagnosis of the disease [11].

Optical diagnosis of cancer is based on laser/light-induced fluorescence, laser Raman spectra, reflectance/scattering spectra, or a combination of these [12-14]. Out of these, analyses based on the native fluorescence of tumor tissue have gained considerable importance since 1924 [15]. While most of the studies were based on fluorescence emission spectra, Alfano et al. employed Stokes shift spectroscopy (SSS) [16, 17]. Extensive studies have been done on tissue characterization, but only a few reports have concerned on the spectral analysis of blood for cancer detection $[18,19]$. Masilamani et al. assume that some of the fluorophores carried by the bloodstream could be excreted through urine as well [20].

Leiner et al. [21] measured the total fluorescence of human urine at various excitation and emission wavelengths and presented it in the 3-dimensional form of contour plots. They observed 3 - 5 distinct fluorescence maxima and made tentative assignments as to the species responsible for the peaks. To date there have been few studies published regarding the 
spectral analysis of urine autofluorescence in cancer patients [22-25]. Our group (Zvarik et al.) found that the ratio of intensities of the peaks at $370 / 440 \mathrm{~nm}$ and at $330 / 420 \mathrm{~nm}$ was 18-times elevated in urine excreted by patients with ovarian cancer as compared to healthy urine samples [24]. Birkova et al. reported another study on ovarian cancer patients, detecting significant differences in several areas of the spectrum between patients with malignant ovarian tumors and healthy women or those with benign histology, but no significant differences in fluorescene intensities between the healthy group and women with benign ovarian tumors [26].

In this study, we investigated the possible cancer screening potential of human urine autofluorescence analysis, its ability to distinguish between benign and malignant ovarian tumors, and the correlation of spectral changes with known prognostic factors - grade and stage.

\section{Patients and methods}

This study was approved by The National Cancer Institute (Slovakia) Ethics Review Board for human studies and registered as FAM OVCA 1. The research was carried out according to the World Medical Association Declaration of Helsinki and all patients signed an informed consent.

Fasting morning urine samples from 42 healthy volunteers, 35 patients with benign ovarian tumors and 36 patients with malignant ovarian tumors were used in this study. All of them were analyzed biochemically for $\mathrm{pH}$, protein, glucose, bilirubin, nitrates, hemoglobin, ketones, acetone, and urobilinogen. The presence of red blood cells, white blood cells, casts, epithelial cells and crystals in urine sediment was also tested in these samples at the Department of Clinical Biochemistry, National Cancer Institute, Bratislava, Slovakia. None of the 42 samples from the healthy volunteers (women aged 26 - 78) showed abnormal laboratory findings and they were referred to as healthy samples, i.e. controls. 36 urine samples were taken from patients with malignant ovarian tumors (women aged 27 - 84) before radical surgery. The histological examination of the removed tissues confirmed malignant ovarian tumors (ICD 10 code C56). 35 urine samples were taken from patients with benign ovarian tumors (women aged $26-84$ ). The histological examination of the removed tissues confirmed benign ovarian tumors (ICD 10 code D27). The demographic characteristics of patients are shown in Table 1. The Body mass index (BMI) was calculated from the formula: $\mathrm{BMI}=\mathrm{W} / \mathrm{H}^{2}$, where $\mathrm{W}$ stands for weight in kilograms and $\mathrm{H}$ stands for height in meters. The Body surface area (BSA) was calculated from the formula of DuBois and DuBois [27]: $\mathrm{BSA}=\left(\mathrm{W}^{0.425} \mathrm{xH}^{0.725}\right) \times 0.007184$. There were no statistically significant differences in BMI, BSA, systolic blood pressure (sBP), diastolic blood pressure ( $\mathrm{dBP}$ ), urine biochemistry and sediment among tested groups. Urine samples were taken before the start of anticancer or antibiotic therapy. They were centrifuged at $3000 \mathrm{rpm}$ for $10 \mathrm{~min}$ at room temperature $\left(22 \pm 1^{\circ} \mathrm{C}\right)$ and undiluted supernatants were used for spectral analysis. The detailed fluorescence data of urine samples were obtained on a LS45 (PerkinElmer) luminescence spectrometer, using the FL WinLab software. Measurements were taken at room temperature $\left(22 \pm 1^{\circ} \mathrm{C}\right)$ in an ultra micro quartz cuvette $(5 \mathrm{~mm}$ excitation path length and $1 \mathrm{~mm}$ emission path length) in $100 \mu \mathrm{l}$ sample volume. Quality control samples including blank ones were run before the beginning of the experiment to ensure the stability of samples and the equipment. Both the emission and excitation slit widths were set to $10 \mathrm{~nm}$. Synchronous fluorescence spectra (SFS) were collected by simultaneously scanning the excitation and emission monochromator in the excitation wavelength range $250-550 \mathrm{~nm}$, with constant wavelength differences $\Delta \lambda=70 \mathrm{~nm}$ between them and in a broad range of urine dilutions $(1: 1-1: 1024)$.

Concentration matrices of synchronous spectra (CMSS) represent the relations between the fluorescence intensity, excitation wavelength and urine dilution (fraction volume). They were created by the set of synchronous fluorescence spectra of urine samples diluted by geometrical progression. The $\mathrm{x}$-axis represents the excitation wavelength of synchronous fluorescence scanning and the $y$-axis the urine dilution (geometric progression). Contour lines connect regions of the same fluorescence intensity and each contour line represents 5 percent of the full fluorescence scale.

To distinguish the diagnostic groups there were 3 class prediction models created from obtained data. The class prediction model 1 (CPM 1) was created from all samples ( $\mathrm{n}=$ 113) to differentiate women with ovarian tumors (both malignant and benign) from healthy women. The class prediction model 2 (CPM 2) included 71 samples from patients with ovarian tumors and was used to distinguish women with malignant and benign tumors (tumor dignity). The last class prediction model 3 (CPM 3) consisted of 78 samples from patients with malignant ovarian tumors and healthy women and was used to distinguish them (presence of malignancy).

Classification of the samples was made using a logistic regression model. Training of the model was carried out using the Weka software, which is a collection of machine learning and statistical tools. The performance of the model was tested by the leave-one-out-cross-validation technique that uses the spectral data of various samples and also the known diagnostic group. The collected data were statistically and graphically processed by the $\mathrm{R}$ software. The relation between the chosen spectral characteristics (intensity and position of peaks at 330 and $360 \mathrm{~nm}$ ) and the prognostic factors (stage, grade) was determined by Kendall`s $\tau$ correlation analysis. We made a box and whisker plot from these spectral characteristics, where the bottom and top of the box are the first and third quartiles, and the band inside the box is the second quartile (the median). The ends of the whiskers represent the lowest value within 1.5 interquartile range (IQR) of the lower quartile, and the highest value within $1.5 \mathrm{IQR}$ of the upper quartile. Any data not included between the whiskers represent outliers (small circles). 
Table 1. The demographic characteristics of patients

\begin{tabular}{|c|c|c|c|}
\hline Patient demographics & $\begin{array}{l}\text { Patients with malignant or } \\
\text { borderline tumors }\end{array}$ & Patients with benign tumors & Healthy women \\
\hline Number of patients (\%) & $36(100 \%)$ & $35(100 \%)$ & $42(100 \%)$ \\
\hline \multicolumn{4}{|l|}{ Age, years } \\
\hline Average & 56,39 & 47,51 & 52,29 \\
\hline Median & 55 & 44 & 54 \\
\hline Range & $27-84$ & $26-84$ & $26-78$ \\
\hline \multicolumn{4}{|l|}{ Race } \\
\hline White (\%) & $36(100 \%)$ & $35(100 \%)$ & $42(100 \%)$ \\
\hline Black (\%) & $0(0 \%)$ & $0(0 \%)$ & $0(0 \%)$ \\
\hline Hispanic (\%) & $0(0 \%)$ & $0(0 \%)$ & $0(0 \%)$ \\
\hline Other (\%) & $0(0 \%)$ & $0(0 \%)$ & $0(0 \%)$ \\
\hline \multicolumn{4}{|l|}{ Nutritional status } \\
\hline Omnivores (\%) & $36(100 \%)$ & $35(100 \%)$ & $42(100 \%)$ \\
\hline Vegetarians (\%) & $0(0 \%)$ & $0(0 \%)$ & $0(0 \%)$ \\
\hline Vegans (\%) & $0(0 \%)$ & $0(0 \%)$ & $0(0 \%)$ \\
\hline \multicolumn{4}{|l|}{ BMI } \\
\hline Average & 27,03 & 25,79 & 24,61 \\
\hline Median & 26,41 & 25,24 & 23,88 \\
\hline Range & $18,76-40,40$ & $18,73-40,75$ & $19,15-38,27$ \\
\hline \multicolumn{4}{|l|}{ BSA } \\
\hline Average & 1,81 & 1,79 & 1,73 \\
\hline Median & 1,83 & 1,74 & 1,72 \\
\hline Range & $1,48-2,24$ & $1,48-2,28$ & $1,45-2,16$ \\
\hline \multicolumn{4}{|l|}{$\mathrm{BP}$} \\
\hline Average & $129,19 / 80,25$ & $130,40 / 78,09$ & $125,20 / 75,83$ \\
\hline Median & $130 / 80$ & $125 / 78$ & $126 / 75$ \\
\hline Range & $95-166 / 55-111$ & $105-170 / 60-100$ & $98-168 / 53-105$ \\
\hline \multicolumn{4}{|l|}{ Tumor histology } \\
\hline serous cystadenoma (\%) & & $17(48,57 \%)$ & \\
\hline mucinous cystadenoma (\%) & & $4(10,26 \%)$ & \\
\hline endometrioid cyst (\%) & & $7(20 \%)$ & \\
\hline dermoid cyst (\%) & & $4(10,26 \%)$ & \\
\hline fibrothecoma (\%) & & $1(2,56 \%)$ & \\
\hline benign monodermal teratoma - struma ovarii (\%) & & $1(2,56 \%)$ & \\
\hline folicular cyst (\%) & & $1(2,56 \%)$ & \\
\hline corpus luteum haemorrhagicum (\%) & & $2(5,13 \%)$ & \\
\hline serous borderline tumor & $5(13,89 \%)$ & & \\
\hline mucinous borderline tumor & $2(5,56 \%)$ & & \\
\hline serous papillary carcinoma & $20(55,56 \%)$ & & \\
\hline mucinous carcinoma & $3(8,33 \%)$ & & \\
\hline endometrioid adenocarcinoma & $1(2,78 \%)$ & & \\
\hline clear cell adenocarcinoma & $3(8,33 \%)$ & & \\
\hline MMMT/carcinosarcoma & $1(2,78 \%)$ & & \\
\hline sarcoma & $1(2,78 \%)$ & & \\
\hline \multicolumn{4}{|l|}{ Tumor differentiation grade } \\
\hline borderline & $7(19,44 \%)$ & & \\
\hline G1 & $1(2,78 \%)$ & & \\
\hline G2 & $5(13,89 \%)$ & & \\
\hline G3 & $22(61,11 \%)$ & & \\
\hline G4 & $1(2,78 \%)$ & & \\
\hline GX & $0(0 \%)$ & & \\
\hline \multicolumn{4}{|l|}{ T stage } \\
\hline T0 & $0(0 \%)$ & & \\
\hline
\end{tabular}


Table 1. (continued)

\begin{tabular}{lcc}
\hline Patient demographics & $\begin{array}{c}\text { Patients with malignant or } \\
\text { borderline tumors }\end{array}$ & Patients with benign tumors \\
\hline T1a & $8(22,22 \%)$ \\
T1b & $1(2,78 \%)$ \\
T1c & $4(11,11 \%)$ \\
T2a & $0(0 \%)$ \\
T2b & $0(0 \%)$ \\
T2c & $0(0 \%)$ \\
T3a & $2(5,56 \%)$ \\
T3b & $3(8,33 \%)$ \\
T3c & $17(47,22 \%)$ \\
TX & $1(2,78 \%)$ \\
N stage & $16(44,44 \%)$ \\
N0 & $5(13,89 \%)$ \\
N1 & $15(41,67 \%)$ \\
NX & \\
M stage & $17(47,22 \%)$ \\
M0 & $4(11,11 \%)$ \\
M1 & $15(41,67 \%)$ \\
MX &
\end{tabular}

BMI - body mass index; BSA - body surface area; sBP - systolic blood pressure; $\mathrm{dBP}$ - diastolic blood pressure

\section{Results}

For the analysis, we chose the shift $\Delta \lambda=70 \mathrm{~nm}$ in synchronous fluorescence spectra, which provides all excitation-emission bands of human urine. From these spectra, CMSS were created in the form of 3D plots (Figure 1), which afforded us complete information in connection with the concentration and fluorescence intensity of sample.

Classification of probands from urine CMSS. We used complex urine CMSS to create CPMs in order to classify probands as healthy individuals, patients with benign and patients with malignant ovarian tumors.

The validation of the CPM 1 (patients with ovarian tumor vs. healthy individuals) gave the number of correctly classified samples (accuracy) 107 (94.69\%) and the number of incorrectly classified samples (inaccuracy) 6 (5.31\%), respectively,

Table 2. Classification of patients with ovarian tumors and healthy individuals

\begin{tabular}{lcc}
\hline & Value & $\begin{array}{c}\text { 95\% confidence } \\
\text { interval (CI) }\end{array}$ \\
\hline Number of patients with ovarian tumors & 71 & - \\
Number of healthy individuals & 42 & - \\
Correctly classified & 107 & - \\
Incorrectly classified & 6 & - \\
Sensitivity & $95.77 \%$ & $88.13 \%-99.07 \%$ \\
Specificity & $92.86 \%$ & $80.49 \%-98.42 \%$ \\
Positive predictive value (PPV) & $95.77 \%$ & $88.13 \%-99.07 \%$ \\
Negative predictive value (NPV) & $92.86 \%$ & $80.49 \%-98.42 \%$ \\
\hline
\end{tabular}

sensitivity $95.77 \%$, specificity $92.86 \%$, positive predictive value (PPV) $95.77 \%$ and negative predictive value (NPV) $92.86 \%$ (Table 2)

The number of correctly and incorrectly classified samples from the CPM 2 (patients with malignant vs. benign ovarian tumor) was $58(81.69 \%)$ and $13(18.31 \%)$, respectively, sensitivity $86.11 \%$, specificity $77.14 \%$, PPV $79.49 \%$ and NPV $84.38 \%$ (Table 3 ).

The number of correctly and incorrectly classified samples in the CPM 3 (patients with malignant tumors vs. healthy

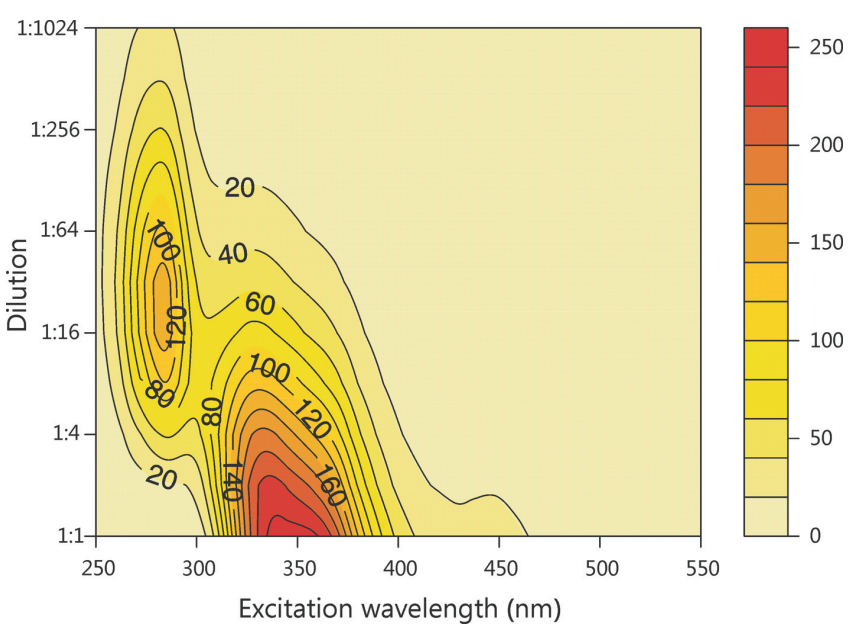

Figure 1. Concentration matrix of synchronous fluorescence spectra $(\Delta \lambda=$ $70 \mathrm{~nm}$ ) of a representative urine sample. 

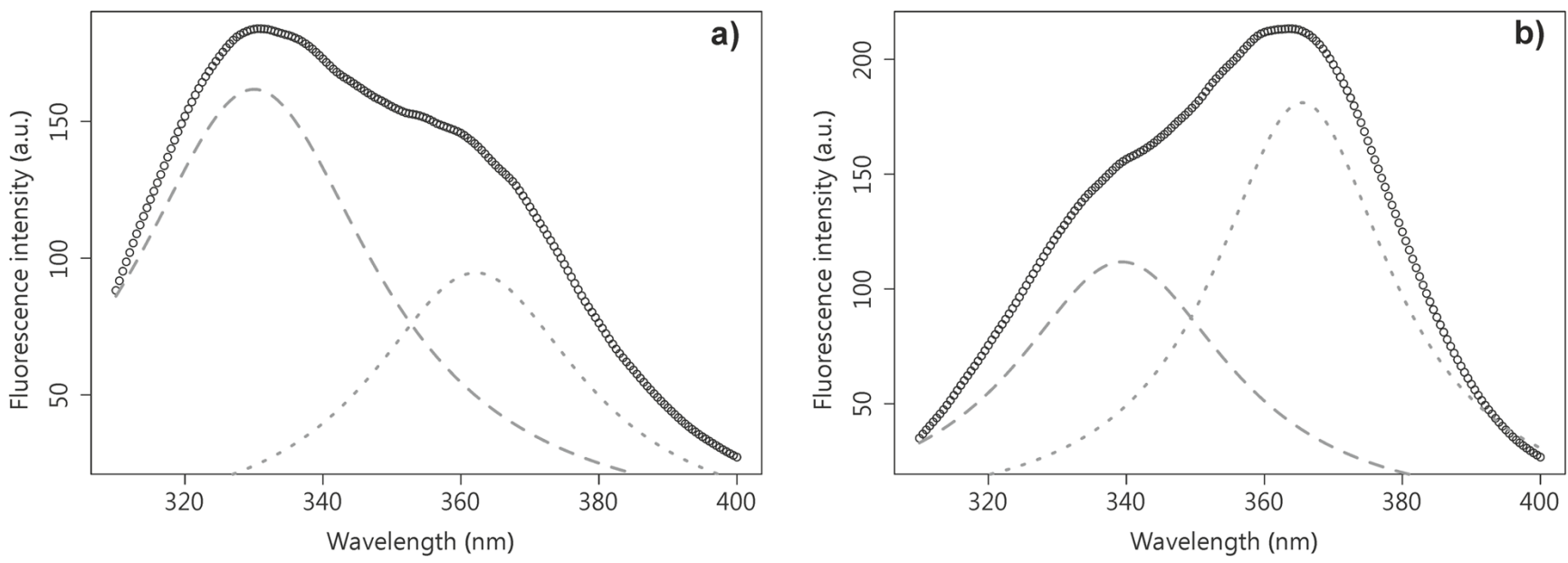

Figure 2. Representative synchronous fluorescence spectrum $(\Delta \lambda=70 \mathrm{~nm})$ of a) urine from healthy individual, b) urine from patient with ovarian tumor. The curve of experimental data (black circles) was mathematically decomposed into two component curves in a process of curve fitting by Lorentzian function. The component curves are peaked at $330 \mathrm{~nm}$ (grey dashed line) and at $360 \mathrm{~nm}$ (grey dotted line), respectively.

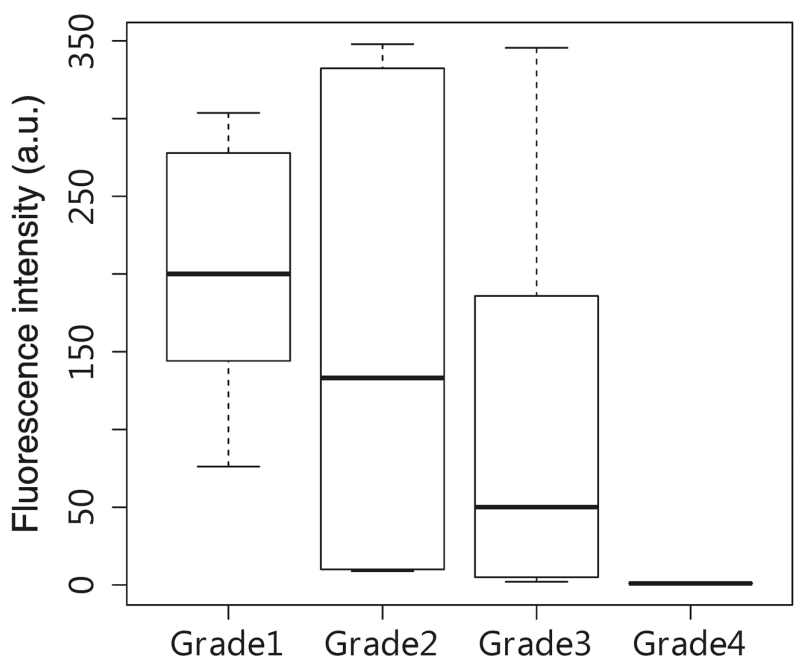

Figure 3. The box and whisker plot of fluorescence intensity at $330 \mathrm{~nm}$ for different ovarian cancer stages $(\tau=-0.33, p<0.02)$.

Table 3. Classification of patients with malignant and benign ovarian tumors

\begin{tabular}{lcc}
\hline & Value & $\begin{array}{c}\mathbf{9 5 \%} \text { confidence } \\
\text { interval (CI) }\end{array}$ \\
\hline Number of patients with malignant tumors & 36 & - \\
Number of patients with benign tumors & 35 & - \\
Correctly classified & 58 & - \\
Incorrectly classified & 13 & - \\
Sensitivity & $86.11 \%$ & $70.49 \%-95.28 \%$ \\
Specificity & $77.14 \%$ & $59.86 \%-89.55 \%$ \\
Positive predictive value (PPV) & $79.49 \%$ & $63.53 \%-90.68 \%$ \\
Negative predictive value (NPV) & $84.38 \%$ & $67.20 \%-94.67 \%$ \\
\hline
\end{tabular}

individuals) was $75(96.15 \%)$ and $3(3.85 \%)$, respectively, sensitivity $91.67 \%$, specificity $100 \%$, PPV $100 \%$ and NPV $93.33 \%$ (Table 4 ).

Correlation of grade and stage with urine fluorescence characteristics. The main difference between healthy individuals and patients with ovarian tumors was observed in synchronous spectra corresponding to the bands at around 330 and $360 \mathrm{~nm}$, which were further used to analyse the intensity and position of peaks (Figure 2). These parameters were also found to correlate with the grade and stage of malignant tumors. Correlation analysis was done exclusively on data from patients with malignant tumors. We observed a significant negative correlation $(\tau=-0.33, p<0.02)$ between the fluorescence intensity of the peak at $330 \mathrm{~nm}$ and the grade (Figure 3). A significant shift of the peak position at $360 \mathrm{~nm}$ to the longer wavelengths with increasing stage (Figure 4a) was detected $(\tau=0.28, p<0.05)$. The correlation of stage and peak position at $330 \mathrm{~nm}$ was on the edge of statistical significance $(\tau=0.28, p=0.0516$, Figure $4 b)$.

Table 4. Classification of patients with ovarian malignant tumors and healthy individuals

\begin{tabular}{lcc}
\hline & Value & $\begin{array}{c}\text { 95\% confidence } \\
\text { interval (CI) }\end{array}$ \\
\hline Number of patients with malignant tumors & 36 & - \\
Number of healthy individuals & 42 & - \\
Correctly classified & 75 & - \\
Incorrectly classified & 3 & - \\
Sensitivity & $91.67 \%$ & $77.51 \%-98.15 \%$ \\
Specificity & $100.00 \%$ & $91.51 \%-100.00 \%$ \\
Positive predictive value (PPV) & $100.00 \%$ & $89.32 \%-100.00 \%$ \\
Negative predictive value (NPV) & $93.33 \%$ & $81.71 \%-98.53 \%$ \\
\hline
\end{tabular}



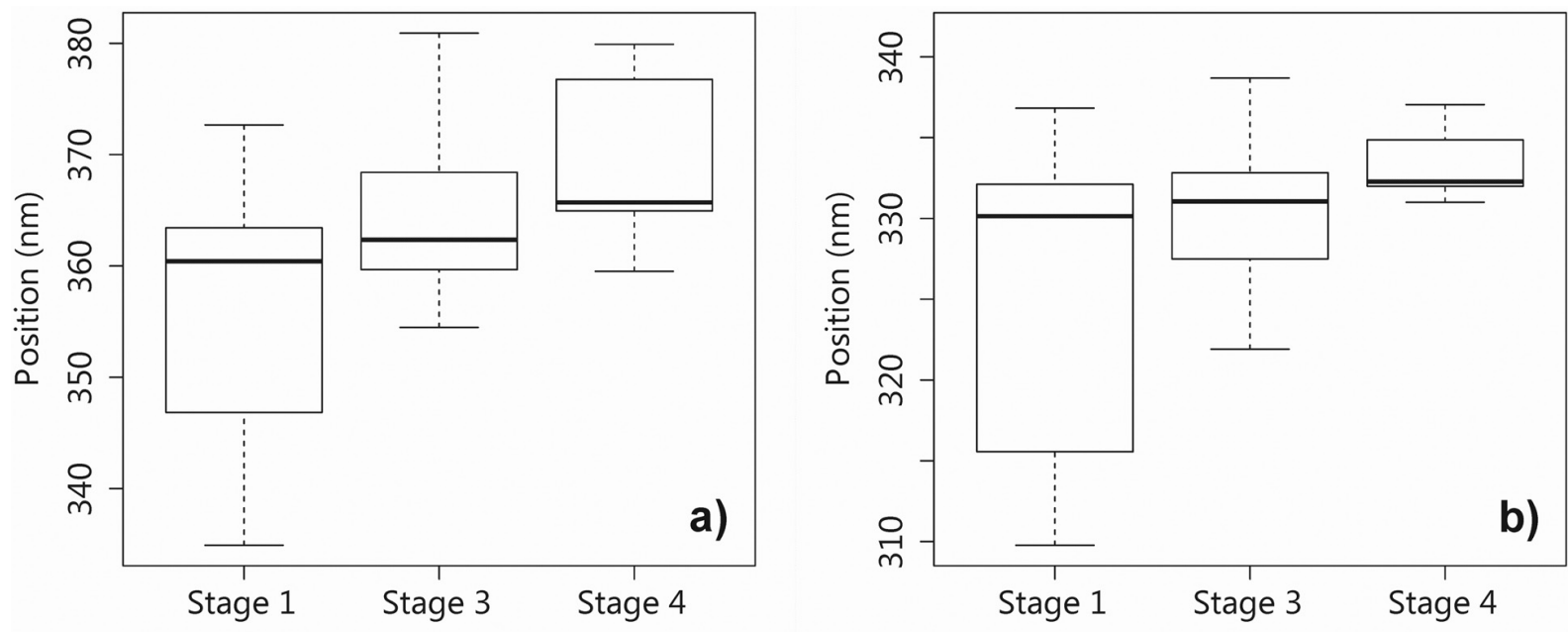

Figure 4. The box and whisker plot of fluorescence peak position at a) $360 \mathrm{~nm}(\tau=0.28, \mathrm{p}<0.05)$ and b) $330 \mathrm{~nm}(\tau=0.28, \mathrm{p}=0.0516)$ for different ovarian cancer stages.

These findings suggest that different fluorophores may prevail at different stages of the disease.

\section{Discussion}

In our study, we examined the screening and diagnostic potential of fluorescence analysis of human urine.

Our classification of patients with ovarian tumors and healthy women (presence of disease) reached high values of both - sensitivity $(95.77 \%, 95 \%$ confidence interval - CI $88.13 \%-99.07 \%)$ and specificity $(92.86 \%$, CI $80.49 \%-$ $98.42 \%$ ) (Table 2). As it was not possible to obtain urine samples from a group of people that would reflect the actual prevalence of the disease in real life (the patients were hospitalized for the disease), obtained PPV and NPV (Table 2) were calculated to the real prevalence of the studied event in the population by the following equations:

$$
\begin{aligned}
P P V & =\frac{\text { Sensitive } * \text { Prevalence }}{\text { Sensitivity } * \text { Prevalence }+(1-\text { Specificity }) *(1-\text { Prevalence })} \\
P P V & =\frac{\text { Specificity } *(1-\text { Prevalence })}{\text { Specificity } *(1-\text { Prevalence })+*(1-\text { Sensitivity }) * \text { Prevalence }}
\end{aligned}
$$

At the estimated prevalence of ovarian tumors $1 \%$ [28] (Table 2), PPV and NPV would be $11.93 \%$ and $99.95 \%$ respectively. Bimanual examination in five studies showed a pooled sensitivity of $45 \%$ (CI $28 \%-68 \%$ ) and specificity of $90 \%$ (CI $80 \%-96 \%$ ) [28], which are lower than our performance indicators. This suggests our approach could be an alternative strategy for adnexal mass detection.

There are several diagnostic methods used to distinguish benign tumors from malignant ones, such as ultrasound (morphology and Doppler) and serum CA-125 levels. Comparing the sensitivity and specificity of ultrasound (sensitivity $86 \%$, CI $79 \%-91 \%$; specificity $91 \%, 80 \%-97 \%$ ), CA- 125 (78\%, CI $75 \%-81 \%$; $78 \%$, CI $71 \%-82 \%$ ) and our classification of patients with malignant and benign ovarian tumors (tumor dignity) (sensitivity $86.11 \%$, CI $70.49 \%-95.28 \%$; specificity $77.14 \%$, CI $59.86 \%-89.55 \%$ ), our approach is comparable to ultrasound in sensitivity and to CA-125 [28] in specificity (Table 3).

Our classification of patients with malignant ovarian tumors and healthy women (presence of malignancy) showed a high sensitivity $(91.67 \%$, CI $77.51 \%-98.15 \%)$, specificity (100\%, CI $91.51 \%-100 \%$ ) (Table 4), PPV $100 \%$ and NPV $93.33 \%$. However, if our PPV and NPV should reflect the actual prevalence of the disease in real life, the PPV and NPV would be $100 \%$ and $99.08 \%$ respectively, at the estimated prevalence of ovarian tumors of $0,1 \%$ [28]. Compared to multimodal screening strategy (MMS) [6] with its sensitivity $89.4 \%$ (CI $76.9 \%-96.5 \%$ ) and specificity $99.8 \%$ (CI $99.8 \%-99.8 \%$ ), our model provides a higher sensitivity and specificity?. An effective screening strategy for ovarian cancer must meet stringent requirements. Owing to the low prevalence of ovarian cancer in the general population, it has been estimated that a sensitivity of more than $75 \%$ and a specificity of $99.6 \%$ for early-stage disease would be required to achieve a PPV of $10 \%$ (ten surgical interventions for each case of malignant disease diagnosed) [29]. As our results do meet the required sensitivity, specificity and PPV, we consider fluorescence analysis of urine to be sufficient for screening purposes.

The proportion of patients who progressed during the 24 months of follow-up in our study was $36.11 \%$. Assuming the 24-months recurrence rate of $36.11 \%$, the PPV and NPV would be $100 \%$ and $95.50 \%$ respectively (Table 4). Based on these results, this method could also be considered as a per- 
spective diagnostic test to detect the recurrence of ovarian cancer after therapy.

From bands in our CMSS, we found meaningful differences in synchronous spectra corresponding to the bands at around 330 and $360 \mathrm{~nm}$ between healthy individuals (Figure $2 \mathrm{a}$ ) and patients with ovarian tumors (Figure $2 \mathrm{~b}$ ). These bands were further used to analyse the intensity and position of peaks of malignant samples in connection with World Health Organization (WHO) grade and Tumor, Node, Metastasis (TNM) stage.

We observed a statistically significant decrease in the fluorescence intensity of the peak excited at $330 \mathrm{~nm}$ with the increasing WHO grade (Figure 3). This finding may be related to the decrease in the concentration of most strongly fluorescing substances excited at this wavelength, i. e. pyridoxic acid and xanthine [21]. The observed reduction of pyridoxic acid as a catabolic product of vitamin B6 is also in agreement with the known association of vitamin B6 and ovarian cancer risk [30]. Xanthine as a product on the pathway of purine degradation can be created from hypoxanthine by xanthine oxidoreductase (XOR). Decreased XOR was found to be associated with poorly differentiated tumors and an abnormal p53 expression, as well as with poorer prognosis in patients with serous ovarian cancer, especially in those with an otherwise more favorable prognostic profile [31]. Moreover, we observed a significant shift in the position of the peak excited at around $360 \mathrm{~nm}$ to the higher wavelengths with the increasing TNM stage (Figure 4a). This revealed spectral shift can be most likely interpreted as a reduction in fluorescence intensity excited at $330 \mathrm{~nm}$, as discussed above, whereas the blue-fluorescing (excited at $\sim 360 \mathrm{~nm}$ ) pteridines and kynurenines become dominant in SFS $[21,24]$ of urine samples from patients with progresive stages of ovarian cancer. A possible explanation for this is the increased degradation of tryptophan, observed in patients with endometrial, ovarian, and vulvar cancer [32]. Reported increased tryptophan degradation compared to controls, resulting in higher serum kynurenine concentrations and a higher kynurenine/tryptophan (kyn/trp) ratio as markers of indoleamine 2,3-dioxygenase (IDO) activity, may be associated with IDO-induced immune escape in these gynecologic cancers. Also pteridine levels in human urine have been reported to be elevated in various types of cancer [33, 34]. Enhanced neopterin biosynthesis during inflammatory states can be caused mainly by macrophages and interferon (IFN)- $\gamma$, but also by other cytokines, such as tumor necrosis factor (TNF)- $\alpha$, and alloantigens [35].

Monitoring of human urine autofluorescence offers an alternative for ovarian cancer screening methods development. This analysis could also find utility as a diagnostic test to detect the recurrence of ovarian cancer after therapy or to select patients with adnexal masses for referral to gynecological oncologists.

Acknowledgements: We are grateful to Assoc. Prof. M. Mego, PhD., Translational Research Unit, National Cancer Institute, Brati- slava, Slovakia, for general support. This work was financially supported by the Scientific Grant Agency of the Ministry of Education, Science, Research and Sport of the Slovak Republic and the Slovak Academy of Sciences VEGA (2/0177/11) and APVV-0134-12.

\section{References}

[1] FERLAY J, SOERJOMATARAM I, ERVIK M, DIKSHIT R, ESER S ET AL. GLOBOCAN 2012 v1.0, Cancer Incidence and Mortality Worldwide: IARC CancerBase No. 11 [Internet]. Lyon, France: International Agency for Research on Cancer; 2013. Available at: http://globocan.iarc.fr. Accessed January 18, 2014.

[2] KOSARY CL. Cancer of the Ovary. In: Ries LAG, Young JL, Keel GE, Eisner MP, Lin YD, Horner, M-J, editors. SEER Survival Monograph: Cancer Survival Among Adults: U.S. SEER Program, 1988 - 2001, Patient and Tumor Characteristics. National Cancer Institute, SEER Program, NIH Pub. No. 07-6215. Bethesda, MD: National Cancer Institute, 2007: 133-144.

[3] BELL R, PETTICREW M, SHELDON T. The performance of screening tests for ovarian cancer: results of a systematic review. Br J Obstet Gynaecol 1998; 105: 1136-1147. http:// dx.doi.org/10.1111/j.1471-0528.1998.tb09966.x

[4] KOBAYASHI H, YAMADA Y, SADO T, SAKATA M, YOSHIDA $S$ et al. A randomized study of screening for ovarian cancer: a multicenter study in Japan. Int J Gynecol Cancer 2008; 18: 414-420. http://dx.doi.org/10.1111/j.1525-1438 .2007.01035.x

[5] PARTRIDGE E, KREIMER AR, GREENLEE RT, WILLIAMS C, XU JL et al. Results from four rounds of ovarian cancer screening in a randomized trial. Obstet Gynecol 2009; 113: 775-782. http://dx.doi.org/10.1097/ $\underline{\text { AOG.0b013e31819cda77 }}$

[6] MENON U, GENTRY-MAHARAJ A, HALLETT R, RYAN A, BURNELL $M$ et al. Sensitivity and specificity of multimodal and ultrasound screening for ovarian cancer, and stage distribution of detected cancers: results of the prevalence screen of the UK Collaborative Trial of Ovarian Cancer Screening (UKCTOCS). Lancet Oncol 2009; 10: 327-340. http://dx.doi. org/10.1016/S1470-2045(09)70026-9

[7] BUYS SS, PARTRIDGE E, BLACK A, JOHNSON CC, LAMERATO L et al. Effect of screening on ovarian cancer mortality: the Prostate, Lung, Colorectal and Ovarian (PLCO) Cancer Screening randomized controlled trial. JAMA 2011; 305: 2295-2303. http://dx.doi.org/10.1001/jama.2011.766

[8] STRASINGER SK, SCHAUB DI LORENZO S. Urinalysis and Body Fluids. Fifth Edition. Philadelphia, PA: F. A. Davis Company; 2001.

[9] VALCARCEL M, GOMEZ-HENS A, RUBIO S, PETIDIER A. Direct quantification of coproporphyrins and uroporphyrinsin urine by derivative synchronous fluorescence spectroscopy. Clin Chem 1987; 33: 1826-1831.

[10] SAUDE EJ, ADAMKO D, ROWE BH, MARRIE T, SYKES BD. Variation of metabolites in normal human urine. Metabol 2007; 3: 439-451. http://dx.doi.org/10.1007/s11306007-0091-1 